\title{
Expected Time to Recruitment in A Single Graded Man Power System With Inter- Decision Times As A Geometric Process
}

\author{
G. Ishwarya, N. Shivaranjani and A. Srinivasan
}

\begin{abstract}
Random depletion of manpower occurs in any marketing organization due to the attrition of personnel when the management takes policy decisions regarding pay, perquisites and targets. This attrition will adversely affect the smooth functioning of the organization in due course of time when the loss of man power is not compensated by recruitment. Frequent recruitment is not advisable as it involves more cost. In view of this situation and from a suitable recruitment policy to plan for recruitment. In this context, for a single graded organization a mathematical model is constructed in this paper using a bivariate recruitment policy based on shock model approach. Assuming that the inter-decision times form a geometric process, the mean time to recruitment is obtained for different cases on the distribution of the thresholds. The influence of nodal parameters on the mean time to recruitment is studied numerically and relevant findings and conclusions are presented.
\end{abstract}

Keywords--- Man Power planning, Shock Models, Geometric Process, Bivariate Recruitment Policy, Mean Time to Recruitment, AMS MSC 2010: 91D35, 91B40, $90 B 70$

\section{INTRODUCTION}

$\mathrm{E}$ XIT of personnel which in other words known as wastage is an important aspect in the study of manpower planning. Many models have been discussed using different kinds of wastages and different types of distributions. Such models could be seen in Bartholomew and Forbes [1] and Bartholomew [2]. The problem of time to recruitment for an organization having one grade is studied using shock model approach by several authors.

For a single graded system, Sathiyamoorthi and Elangovan [13] Sathiyamoorthi and Parthasarathy[14] Parthasarathy and Vinod [11] Muthaiyan and Sathiyamoorthi [9] Kasturri [7] Uma [16] Esther Clara and Srinivasan [4] have obtained the performance measures namely mean and variance of the time to recruitment using a univariate CUM policy of recruitment for different distributions of the threshold for the loss of man power in the organization and for independent and correlated

G. Ishwarya, Assistant Professor in Mathematics, Agni College of Technology, Thalambur, Chennai.E-mail: mathishwarya@gmail.com

N. Shivaranjani, Department of Mathematics, Bishop Heber College, Trichy.

Dr. A. Srinivasan, Department of Mathematics, Bishop Heber College, Trichy. E-mail: mathsrinivas@yahoo.co.in inter-decision times. The problem of time to recruitment is studied using a univariate Max policy of recruitment by Muthulakshmi [10] Venkatesh [18] Poorni Perianayaki [12].Komathi [8] has studied the results of Poorni Perianayaki [12] using a bivariate CUM policy of recruitment. For a single graded system Esther Clara and Srinivasan ([5], [6]) have studied the problem of time to recruitment using a bivariate CUM policy of recruitment involving optional and mandatory thresholds when the inter-decision times are (i) independent and identically distributed exponential random variables (ii) correlated random variables. Sudha [15] Venkat Lakshmi [19] Brindha [3] Komathi [8] have obtained the mean time to recruitment under different conditions using bivariate Max policy of recruitment. Recently Muthaiyan [9] has estimated the expected time to recruitment for a single graded manpower system when the inter-decision times form an order statistics using a univariate CUM policy of recruitment. This work has been studied by Uma et al., [17] using a bivariate recruitment policy. The main objective of this paper is to study the work of Uma et al., [17] using a bivariate CUM policy of recruitment when the inter-decision times form a geometric process. This assumption on the inter-decision times is more realistic since the monotonicity of the inter-decision times which is in built in the geometric process is not taken into account in the earlier work. This paper is organized as follows. In section 2 model descriptions is given and the analytical expression for the mean time to recruitment is obtained for different cases on the distribution of the threshold for the loss of manpower in the organization. In section 3 the analytical results are numerically illustrated, reporting the relative changes in the expected time to recruitment and relevant conclusions.

\section{MODEL DESCRIPTION AND ANALYSIS}

The following assumptions and notations characterize the model under consideration.

\section{Assumption:}

1. An organization takes decisions at random epochs in $(0, \infty)$ and at every decision making epoch a random number of persons quit the organization.

2. There is an associated loss of manpower to the organization if a person quits.

3. The loss of manpower process forms a sequence of independent and identically distributed random variables with parameter $\lambda$.

4. Recruitment is made if the cumulative loss of manpower crosses a threshold level or the number of 
decisions taken crosses its threshold, whichever is earlier. This is the bivariate recruitment policy employed in this paper.

5. The wastage of manpower on the successive decision epochs is linear and cumulative.

6. The inter-decision times form a geometric process with parameter ' $a$ '.

7. The loss of manpower process and the process of inter-decision times are statistically independent.

\section{Notations:}

$\mathrm{X}_{\mathrm{i}}$ : a random variable denoting the loss of manpower due to the $\mathrm{i}^{\text {th }}$ decision epoch, $\mathrm{i}=1,2,3, \ldots$ with p.d.f $\mathrm{g}($.$) or p.g.f \varphi($. according as $X_{i}$ is continuous or discrete

$\mathrm{S}_{\mathrm{k}}$ : the cumulative loss of manpower in the first k-decisions with p.d.f $\mathrm{g}_{\mathrm{k}}($.$) and c.d.f \mathrm{G}_{\mathrm{k}}($.

$\bar{g}($.$) : Laplace transform of \mathrm{g}($.)

$\mathrm{U}_{\mathrm{k}}$ : a continuous random variable denoting the time between $(\mathrm{k}-1)^{\text {th }}$ and $\mathrm{k}^{\text {th }}$ decisions, $\mathrm{k}=1,2,3 \ldots$, with p.d.f $\mathrm{f}($.$) and c.d.f$ $\mathrm{F}($.)

$\mathrm{Y}$ : a continuous random variable denoting the threshold for the loss of manpower in the organization

$\mathrm{d}$ : a positive integer denoting the threshold for the number of decisions

$\mathrm{T}$ : a continuous random variable denoting the time to recruitment with p.d.f $\ell$ (.)

$\mathrm{V}_{\mathrm{k}}(\mathrm{t})$ : probability that there are exactly $\mathrm{k}$ - decisions taken in $(0, \mathrm{t}]$

$\mathrm{E}(\mathrm{T})$ : Mean Time To Recruitment.

Main Result:

$\mathrm{P}[\mathrm{T}>\mathrm{t}]=$

$\sum_{k=0}^{d-1}\left\{\begin{array}{l}\text { Probability that exactly } \mathrm{k} \text { - decisions are } \\ \text { taken in }(0, \mathrm{t}] \text { and the cumulative loss of } \\ \text { man power does not cross the } \\ \text { threshold level }\end{array}\right\}$

(i.e.) $\mathrm{P}[\mathrm{T}>\mathrm{t}]=\sum_{k=0}^{d-1} \mathrm{~V}_{\mathrm{k}}(\mathrm{t}) \mathrm{P}\left(\mathrm{S}_{\mathrm{k}}<\mathrm{Y}\right)$

Since $\left\{U_{k}\right\}$ is a geometric process it is known that

$\overline{f_{k}}(s)=\prod_{n=1}^{k} \bar{f}\left(\frac{s}{a^{n-1}}\right)$

From renewal theory (Medhi (1994)), it is known that $\mathrm{V}_{\mathrm{k}}(\mathrm{t})=\mathrm{F}_{\mathrm{k}}(\mathrm{t})-\mathrm{F}_{\mathrm{k}+1}(\mathrm{t})$ with $\mathrm{F}_{0}(\mathrm{t})=1$.

We now obtain the p.d.f $\ell(\mathrm{t})$ and the mean time to recruitment by considering different cases for the distribution of Y.

Case (i): $\mathrm{Y} \sim \operatorname{Exp}(\mu)$

In this case it can be shown that

$\mathrm{P}(\mathrm{T}>\mathrm{t})=1+\sum_{k=1}^{d-1} \mathrm{~F}_{\mathrm{k}}(t)(\overline{\mathrm{g}}(\mu))^{\mathrm{k}-1}[(\bar{g}(\mu))-1]$ $\ell(\mathrm{t})=\frac{d}{d t}[1-\mathrm{P}(\mathrm{T}>\mathrm{t})]=(1-\bar{g}(\mu)) \sum_{k=1}^{d-1} \mathrm{f}_{\mathrm{k}}(t)(\overline{\mathrm{g}}(\mu))^{\mathrm{k}-1}$

$\bar{\ell}(\mathrm{s})=(1-\bar{g}(\mu)) \sum_{k=1}^{d-1}\left[\prod_{n=1}^{k} \bar{f}\left(\frac{s}{a^{n-1}}\right)(\bar{g}(\mu))^{k-1}\right]$

Since $\mathrm{E}(\mathrm{T})=\left[-\frac{d}{d s}(\bar{\ell}(s))\right]_{s=0}$ and $\bar{g}(\mu)=\frac{\lambda}{\lambda+\mu}$, from (5) it can be shown that

$\mathrm{E}(\mathrm{T})=$

$\frac{a E\left(U_{1}\right)}{a-1}\left[1-\left(\frac{\lambda}{\lambda+\mu}\right)^{d-1}\right]-\frac{a \mu E\left(U_{1}\right)}{(a-1)(a(\lambda+\mu)-\lambda)}\left[1-\left(\frac{\lambda}{a(\lambda+\mu)}\right)^{d-1}\right]$

(6) gives the mean time to recruitment for case (i)

Case (ii): Y follows an extended exponential distribution with scale parameter $\mu$ and shape parameter 2

In this case, $\mathrm{P}(\mathrm{Y} \leq \mathrm{x})=\left(1-\mathrm{e}^{-\mu \mathrm{x}}\right)^{2}$

As in case (i), it can be shown that,

$\mathrm{P}(\mathrm{T}>\mathrm{t})=2\left[1+\sum_{k=1}^{d-1} \mathrm{~F}_{\mathrm{k}}(t)(\overline{\mathrm{g}}(\mu))^{\mathrm{k}-1}[(\bar{g}(\mu))-1]\right]-$

$$
\left[1+\sum_{k=1}^{d-1} \mathrm{~F}_{\mathrm{k}}(t)(\overline{\mathrm{g}}(2 \mu))^{\mathrm{k}-1}[(\bar{g}(2 \mu))-1]\right]
$$

$\ell(\mathrm{t})=\frac{d}{d t}(1-\mathrm{P}(\mathrm{T}>\mathrm{t}))=2\left[(1-\bar{g}(\mu)) \sum_{k=1}^{d-1} \mathrm{f}_{\mathrm{k}}(t)(\overline{\mathrm{g}}(\mu))^{\mathrm{k}-1}\right]-$

$$
(1-\bar{g}(2 \mu)) \sum_{k=1}^{d-1} \mathrm{f}_{\mathrm{k}}(t)(\overline{\mathrm{g}}(2 \mu))^{\mathrm{k}-1}
$$

and

$$
\begin{aligned}
& \bar{\ell}(s)=2\left[(1-\bar{g}(\mu)) \sum_{k=1}^{d-1}\left[\prod_{n=1}^{k} \bar{f}\left(\frac{s}{a^{n-1}}\right)(\bar{g}(\mu))^{k-1}\right]\right]- \\
& (1-\bar{g}(2 \mu)) \sum_{k=1}^{d-1}\left[\prod_{n=1}^{k} \bar{f}\left(\frac{s}{a^{n-1}}\right)(\bar{g}(2 \mu))^{k-1}\right]
\end{aligned}
$$

Since $\mathrm{E}(\mathrm{T})=\left[-\frac{d}{d s}(\bar{\ell}(s))\right]_{s=0}$ and $\bar{g}(\mu)=\frac{\lambda}{\lambda+\mu}$, from (7) it can be shown that 


$$
\begin{aligned}
E(T)=2 & {\left[\begin{array}{l}
\frac{a E\left(U_{1}\right)}{a-1}\left[1-\left(\frac{\lambda}{\lambda+\mu}\right)^{d-1}\right]- \\
\frac{a \mu E\left(U_{1}\right)}{(a-1)(a(\lambda+\mu)-\lambda)}\left[1-\left(\frac{\lambda}{a(\lambda+\mu)}\right)^{d-1}\right]
\end{array}\right] } \\
& {\left[\begin{array}{l}
\frac{a E\left(U_{1}\right)}{a-1}\left[1-\left(\frac{\lambda}{\lambda+2 \mu}\right)^{d-1}\right]- \\
{\left[\frac{2 a \mu E\left(U_{1}\right)}{(a-1)(a(\lambda+2 \mu)-\lambda)}\right.} \\
{\left[1-\left(\frac{\lambda}{a(\lambda+2 \mu)}\right)^{d-1}\right]}
\end{array}\right] }
\end{aligned}
$$

(10) gives the mean time to recruitment for case (ii).

Case (iii): $\mathrm{X}_{\mathrm{i}} \sim$ Poisson $(\lambda)$; $\mathrm{Y} \sim \mathrm{Geo}(\mu)$

Proceeding as in case (i), it can be shown that

$$
\begin{aligned}
& \ell(\mathrm{t})=[1-\varphi(\bar{\mu})] \sum_{k=1}^{d-1} f_{k}(t)(\varphi(\bar{\mu}))^{k-1} \\
& \bar{\ell}(s)=(1-\varphi(\bar{\mu})) \sum_{k=1}^{d-1}\left[\prod_{n=1}^{k} \bar{f}\left(\frac{s}{a^{n-1}}\right)(\varphi(\bar{\mu}))^{k-1}\right]
\end{aligned}
$$

where $\varphi($.$) is the p.g.f. of \mathrm{X}_{\mathrm{i}}$.

$$
\begin{aligned}
& E(T)=\frac{a E\left(U_{1}\right)}{a-1}\left(1-\left(e^{-\lambda \mu}\right)^{d-1}\right)- \\
& \frac{\left(1-e^{-\lambda \mu}\right) E\left(U_{1}\right)}{(a-1) a^{d-2}\left(a-e^{-\lambda \mu}\right)}\left(a^{d-1}-\left(e^{-\lambda \mu}\right)^{d-1}\right)
\end{aligned}
$$

(13) gives the mean time to recruitment for case (iii).

\section{NUMERICAL ILLUSTRATION}

The mean time to recruitment is numerically illustrated for all the three cases by varying one parameter and keeping the other parameters fixed. The effect of the nodal parameters $\lambda$ and $\theta$ on the mean time to recruitment is shown in table 1.The impact of ' $a$ ' when $a>1$ on the mean time is shown in table 2 .

Table 1:Effect of $\lambda$ and $\theta$ on the Expected Time to

Recruitment $(\mathrm{d}=20 ; \mathrm{a}=5 ; \mu=1.0)$

\begin{tabular}{|c|c|c|c|c|}
\hline \multirow{2}{*}{$\lambda$} & \multirow{2}{*}{$\theta$} & \multicolumn{3}{|c|}{$\mathrm{E}(\mathrm{T})$} \\
\cline { 3 - 5 } & & Case(i) & Case (ii) & Case(iii) \\
\hline 0.5 & 1.5 & 0.7143 & 0.7342 & 0.7585 \\
1.0 & 1.5 & 0.7407 & 0.7672 & 0.7195 \\
1.5 & 1.5 & 0.7575 & 0.7860 & 0.6978 \\
2.0 & 1.5 & 0.7688 & 0.7970 & 0.6852 \\
\hline 1.5 & 0.2 & 5.6818 & 5.8955 & 4.9417 \\
1.5 & 0.5 & 2.2725 & 2.3577 & 2.0934 \\
1.5 & 1.0 & 1.1363 & 1.1790 & 1.0467 \\
1.5 & 1.5 & 0.7575 & 0.7860 & 0.6978 \\
1.5 & 2.0 & 0.5680 & 0.6250 & 0.5234 \\
\hline
\end{tabular}

Table 2: Effect of 'a' on the Expected Time to Recruitment $(\mathrm{d}=20 ; \mu=1.0 ; \lambda=1.0 ; \theta=1.5)$

\begin{tabular}{|c|c|c|c|}
\hline $\mathrm{a}$ & Case(i) & Case(ii) & Case(iii) \\
\hline 2 & 0.9999 & 1.1998 & 0.8168 \\
3 & 0.8461 & 0.9423 & 0.7597 \\
4 & 0.7979 & 0.8686 & 0.7340 \\
5 & 0.7407 & 0.7672 & 0.6852 \\
& & & \\
\hline
\end{tabular}

From the above tables, we made the following conclusion:

1. When $\lambda$ increases and keeping other parameters fixed, the mean time for recruitment increases for cases (i) and (ii) and decreases for case (iii).In fact when $\lambda$ increases, the average loss of manpower decreases for cases (i) and (ii) and increases for case (iii) and hence the corresponding conclusions on $\mathrm{E}(\mathrm{T})$ are realistic.

2. The mean time for recruitment decreases as increases, keeping other parameters fixed. This conclusion is also realistic since as increases, the decisions are taken frequently on the average which in turn advances the time to recruitment on the average.

3. For $a>1$, the mean time for recruitment decreases as ' $a$ ' increases, keeping other parameters fixed. In fact, when a>1 the geometric process of inter-decision times is stochastically decreasing and hence the same conclusion in 2 is also reflected in this case.

\section{CONCLUSION}

The mean time to recruitment is obtained for different cases on the distribution of the thresholds by assuming that the inter-decision times form a geometric process. The influence of nodal parameters on the mean time to recruitment is also studied numerically. On seeing all the three cases, from the organization point of view, case (ii) is more suitable for the organization to postpone their recruitment, since case (ii) has the maximum $\mathrm{E}(\mathrm{T})$ out off all the three.

\section{REFERENCES}

[1] Bartholomew D.J and Forbes A.F, "Statistical techniques for manpower planning", John Wiley and Sons, New York, 1979.

[2] Bartholomew D.J, "Statistical models for social processes" (3rd Ed.,), John Wiley and Sons, New York, 1973.

[3] Brindha M, "Stochastic model on the time to recruitment in a single graded manpower system associated with a bivariate policy of recruitment", M.Phil., Dissertation, Bharathidasan University, 2008.

[4] Esther Clara J.B and Srinivasan A, "Expected time for recruitment in a single graded manpower system with two thresholds" ,Proceedings of the AICTE sponsored National Conference on Recent Developments and Applications of Probability Theory, Random Process and Random Variables in Computer Science, Pp. 98-102, 2008

[5] Esther Clara J.B and Srinivasan A, "A stochastic model for the expected time to recruitment in a single graded manpower system with two thresholds using bivariate policy", Recent Research in Science and Technology, Vol. 2, Pp. 70-75, 2010.

[6] Esther Clara J.B and Srinivasan A, "A stochastic model for the expected time to recruitment using bivariate recruitment policy involving two thresholds associated with correlated inter-decision times", International 
Journal of Mathematical Sciences and Engineering Applications, Vol. 5(III), Pp. 281-288, 2011.

[7] Kasturri K, "Mean time for recruitment and cost analysis on some univariate policies of recruitment in manpower models", Ph.D. Thesis, Bharathidasan University, 2007.

[8] Komathi S, "Stochastic models on the time for recruitment in a single graded manpower system with correlated inter-decision times using a bivariate policy of recruitment", M.Phil., Dissertation Bharathidasan University, 2008.

[9] Muthaiyan A, "A study on stochastic models in manpower planning", Ph.D. Thesis, Bharathidasan University, 2010.

[10] Muthulakshmi N, Srinivasan A and Kasturri K, "Mean and variance of the time for recruitment in a single graded manpower system with an univariate policy of recruitment involving geometric threshold", Acta Ciencia Indica, Vol. 34, Pp.1637-1641, 2008.

[11] Parthasarathy $S$ and Vinoth R, "Determination of expected time to recruitment in manpower planning", Recent Research in Science and Technology, Vol. 1(3), Pp. 147-150, 2009.

[12] Poorni Perianayaki S.N, "Mean and variance of the time to recruitment in single graded manpower system having correlated inter-decision times associated with an univariate policy of recruitment", M.Phil., Dissertation, Bharathidasan University, 2007.

[13] Sathiyamoorthi $R$ and Elangovan R, "Shock model approach to determine the expected time for recruitment", Journal of Decision and Mathematika Sciences, Vol. 3(1-3), Pp. 67-78, 1998.

[14] Sathiyamoorthi R and Parthasarathy S, "On the expected time to recruitment in a two graded marketing organization", Indian Association for Productivity Quality and Reliability, Vol. 27(1), Pp. 77-81, 2002.

[15] Sudha A, Saavithri V and Srinivasan A, "Mean and variance of the time for recruitment in a single graded manpower system associated with a bivariate policy of recruitment", Acta Ciencia Indica, Vol. 34M(1), Pp. 85-89, 2008.

[16] Uma K.P, "A study on manpower models with univariate and bivariate policies of recruitment", Ph.D. Thesis, Avinashilingam University for Women, 2010.

[17] Uma K.P, Shivarajani $N$ and Srinivasan A, "Expected time to recruitment in a single graded man power system with inter-decision times as an order statistics", To appear in the Proceedings of Heber International Conference on Applied Mathematics and Statistics, 2012.

[18] Venkatesh B, "Mean and variance of the time to recruitment for a single graded manpower system associated with an univariate policy of recruitment", M.Phil Dissertation Bharathidasan University, 2007.

[19] Venkat Lakshmi K, "Mean and variance of the time to recruitment for a single graded manpower system associated with a bivariate policy of recruitment", M.Phil., Dissertation Bharathidasan University, 2007. 